\title{
Visualization of the Process of a Nanocarrier- Mediated Gene Delivery: Stabilization, Endocytosis, Endosomal Escape and Exocytosis of Genes for Intercellular Spreading
}

\section{Zhongzheng Ma}

China Agricultural University College of Plant Protection

Yang Zheng

Yangzhou University

\section{Zijian Chao}

China Agricultural University College of Plant Protection

\section{Hongtao Chen}

Beijing University of Chemical Technology

\section{Yunhui Zhang}

China Agricultural University College of Plant Protection

\section{Meizhen Yin}

Beijing University of Chemical Technology

\section{Jie Shen}

China Agricultural University College of Plant Protection

Shuo Yan ( $\triangle$ yanshuo2011@foxmail.com )

China Agricultural University College of Plant Protection https://orcid.org/0000-0001-9685-9810

\section{Short Report}

Keywords: Cellular uptake, Clathrin, Endocytosis, dsRNA, Nanocarrier, RNA interference

Posted Date: January 20th, 2022

DOl: https://doi.org/10.21203/rs.3.rs-1255599/v1

License: (c) (i) This work is licensed under a Creative Commons Attribution 4.0 International License. Read Full License 


\section{Abstract}

Nanoparticles have been widely applied as gene carrier for improving RNA interference (RNAi) efficiency in medical and agricultural fields. However, the mechanism and delivery process of nanoparticlemediated RNAi is not directly visualized. Here we synthesized a star polymer (SPc) consisted of a hydrophilic shell with positively-charged tertiary amine in the side chain as a gene nanocarrier, which was taken as an example to investigate the mechanism in gene delivery. The SPc could assemble with dsRNA spontaneously through electrostatic force, hydrogen bond and van der Waals force. Interestingly, the SPc could protect dsRNA from degradation by RNase A, insect hemolymph and immune cells, thus remarkably increasing the stability of dsRNA. Meanwhile, the SPc could efficiently promote the cellular uptake, endosomal escape and active exocytosis for systematic spreading of dsRNA. Transcriptome analysis revealed that the SPc could up-regulate some key genes such as Chc, AP2S1 and Arf 1 for activating clathrin-mediated endocytosis. Furthermore, the suppression of endocytosis by a specific inhibitor hindered the cellular uptake of SPc-delivered dsRNA in vitro, and the subsequent RNAi effect was also disappeared in vivo. To our knowledge, our study is the first direct visualization of the detailed cellular delivery process and mechanism of nanocarrier-mediated gene delivery. Above mechanism supports the application of nanocarrier-based RNAi in gene therapy and pest management.

\section{Introduction}

RNA interference (RNAi) functions via post-transcriptional gene silencing and is highly conserved in eukaryotic organisms. RNAi technique is widely used to interfere gene function and shows a great potential for gene therapy and pest management in medical and agricultural fields. ${ }^{1-6}$ Gene therapy is a promising tool for the treatment of several diseases, including cancer, central nervous system disorders and metabolic disorders, but the major limitation of small interfering RNA (siRNA) or double-stranded RNA (dsRNA) is the inability of naked siRNA/dsRNA to passively diffuse through cellular membrane due to the electrostatic repulsion from the anionic cell membrane surface ${ }^{7-10}$ Meanwhile, siRNA/dsRNA is highly susceptible to nuclease-induced degradation and thus their therapeutic actions are limited. ${ }^{8,11}$ Similar to the application in medical field, RNAi has also been recognized as a novel and safe strategy in pest green management. ${ }^{6,12-15}$ However, RNAi efficiency varies greatly among different insect species, and the major limitations for efficient RNAi include dsRNA instability, low efficiency of dsRNA cellular internalization, deficient core RNAi machinery and impaired systemic spreading of dsRNA, which constrains the application of RNAi-based gene therapy and pest management. ${ }^{16-21}$

Nano-delivery systems for drugs and other bioactive molecules in medical and agricultural fields have been bloomed over the past 30 years. ${ }^{22,23}$ Several types of synthetic gene vectors such as cationic lipids, chitosan and quantum dots have been designed and constructed to overcome the delivery obstacle for efficient RNAi. ${ }^{24-27}$ Nanocarrier-mediated dsRNA delivery was firstly described in the silencing of chitin synthase genes in African malaria mosquito (Anopheles gambiae), and the chitin synthase 1 transcript level and chitin content were reduced by $62.8 \%$ and $33.8 \%$ in the larvae fed on chitosan-delivered 
dsRNA. ${ }^{28}$ Subsequently, the nanocarrier-mediated RNAi was tested in several insect species, and high RNAi efficiency has been observed in fall armyworm Spodoptera frugiperda, ${ }^{29}$ Yellow fever mosquito Aedes aegypti, ${ }^{30}$ beet armyworm S. exigua ${ }^{31}$ and German cockroach Blattella germanica ${ }^{32}$. Thus, the nanoparticle-mediated RNAi has a good potential in pest sustainable control, but the detailed delivery process and mechanism of nanoparticle-mediated RNAi have not been directly visualized.

Previous studies have implied that the application of nano-delivery system might solve the systemic and intracellular barriers such as rapid degradation and poor cellular uptake. ${ }^{33-36}$ Nanoparticles, such as polymers and cationic lipids, may protect dsRNA molecules from enzymatic degradation and improve their stability in various insect tissues. ${ }^{31,37}$ On the other hand, nanoparticles may promote the translocation of dsRNA across cell membranes, thus improving dsRNA delivery. Based on current knowledge, receptor-mediated endocytosis may be the major route for nanocarriers to enter into the cells. ${ }^{38-40}$ Nanocarriers are usually coated by membrane-bound vesicles called endosomes following cellular uptake, and the late endosomes ultimately fuse with degradative lysosomes. ${ }^{41,42}$ Therefore, endosomal escape has become another key step for nano-delivery systems. ${ }^{43}$ Although there are some studies focusing on nanoparticle-mediated dsRNA delivery in therapeutic applications, the mechanism for nanoparticle-mediated RNAi should be further directly visualized.

Our group has constructed a nanocarrier-based platform for pest management to deliver various pesticidal substances, such as dsRNA ${ }^{44,45}$, insect virus DNA ${ }^{46}$, narrow-spectrum pesticides ${ }^{47}$ and Bt toxins ${ }^{48}$. To decrease the production cost of nanoparticles for field application, a facile-synthesized star polycation (SPc) is designed to construct a transdermal dsRNA delivery system for controlling soybean aphids, which has been selected for Research Highlights from China collection by Springer Nature ${ }^{49,50}$. Subsequently, the SPc-delivered dsRNA is applied to disrupt the wing development of fruit flies and green peach aphids and inhibit the feeding behavior of oriental fruit moths ${ }^{51-53}$. So far, the SPc has been widely shared with researchers in China, and the transdermal dsRNA delivery system has been successfully applied in more than 30 insect species. The current study focused on the mechanism and delivery process of nanoparticle-mediated RNAi. We took SPc as an example, determined the interaction of SPc with dsRNA, tested the stability of SPc-complexed dsRNA, investigated the cellular uptake and intracellular fate of dsRNA/SPc complex, and finally confirmed the major gene pathway for the cellular uptake of dsRNA/SPc complex. Our study directly visualized the detailed cellular process and mechanism of polymer-mediated RNAi, which supports the development and practice of RNAi-based gene therapy and pest management.

\section{Results And Discussion}

\section{Loading Capacity of SPc and Its Interaction with dsRNA}

The SPc was designed and constructed as a gene vector for efficient dsRNA delivery, achieving good gene silencing effects on insects. ${ }^{49-53}$ However, the interactions between SPc and dsRNA were not very 
clear. To investigate the loading capacity of SPc toward dsRNA, dseGFP was mixed with SPc at various mass ratios and analyzed using gel retardation test. As shown in Fig. $1 \mathrm{~A}$, the band's intensity of the migrated dseGFP gradually decreased with the increasing mass ratios, indicating that the SPc had excellent performance in combining with dsRNA. The SPc is consisted of a hydrophilic shell with positively-charged tertiary amine in the side chain. We deduced that the tertiary amine could combine with negatively-charged nucleic acids through electrostatic interaction, which resulted in the electronegativity loss of dsRNA.

ITC was then performed to further illustrate the interaction between dsRNA and SPc (Fig. 1B). A high affinity constant $\left(K_{a}\right)$ of $5.526 \times 10^{7} \mathrm{M}^{-1}$ and a low dissociation constant $\left(K_{d}\right)$ of $1.810 \times 10^{-8} \mathrm{M}$ indicate that there is an effective and strong interaction between SPc and dseGFP, and this interaction is automatic due to the negative $\Delta \mathrm{G}$ of $-43.9 \mathrm{KJ} / \mathrm{mol}$. The negative $\Delta \mathrm{H}$ and $\Delta \mathrm{S}$ suggest that the hydrogen bond and van der Waals force also play an important role in the self-assembly of dseGFP/SPC complex. ${ }^{54}$ Based on the chemical structure, multifunctional SPc can be complexed with exogenous substances through various interaction forces such as hydrogen bond and van der Waals force with chitosan, electrostatic interaction with eugenol, thiamethoxam and osthole, and hydrophobic interaction with matrine. ${ }^{55-58}$ Different self-assembly mechanisms of SPc with exogenous substances are beneficial for expanding the application area of SPC.

\section{Efficient Protective Effects of Nanocarriers on dsRNA}

The degradation of dsRNA is very fast, ${ }^{59}$ which was also confirmed by our results that the dseGFP could be degraded quickly by RNase A (Fig. 2A). Interestingly, there was no significant change in band density of SPc-complexed dseGFP treated with RNase A (Fig. 2B-B'). The SPc-complexed dseGFP was purified and quantified, and the results demonstrated that RNase A could not degrade SPc-loaded dsRNA (Fig. 2C). Similarly, the SPc could also prevent dseGFP from degradation by hemolymph, providing a strong protection (Fig. S1). Meanwhile, a perylenediimide-cored cationic dendrimer (G2) previously constructed by our group was also tested in the current study. ${ }^{60}$ The stability of G2-complexed dsRNA was also improved remarkably (Fig. S2 and S3). Similar to previous studies, a guanidine-containing polymer is able to protect dsRNA against nucleolytic degradation at $\mathrm{pH} 11$ in gut juice of beet armyworms for up to 30 h. ${ }^{31}$ Cationic lipids can protect dsRNA from degradation by endonuclease present in Sf9 cells conditioned medium, hemolymph and midgut lumen contents collected from the larvae of S. frugiperda. ${ }^{37}$

In addition to the fast digestion of free dsRNA by dsRNases, immune cells in hemolymph also attack exotic dsRNA. ${ }^{61,62}$ Fluorescent dseGFP was synthesized for the stability test of nanocarrier-complexed dsRNA in confrontation with immune cells. The combination of $\mathrm{G} 2$ with dseGFP changed the fluorescence intensity of both dseGFP and G2, and the addition of SPc did not lead to such effects, suggesting that the SPc is fit for investigating the fate of nanocarrier-delivered fluorescent dsRNA in immune cells (Fig. S4). Fluorescent signal was strongly detectable in immune cells treated with fluorescent dseGFP/SPc complex (Fig. 2E-E'), whereas the signal of naked dseGFP was undetectable in 
immune cells (Fig. 2D-D'). Our results indicate that the dsRNA/SPc complex could remain relatively stable in immune cells of $S$. frugiperda.

\section{SPc-Mediated Efficient Delivery of dsRNA}

Previous study has confirmed that the dsRNA could be taken up by Sf9 cells within 30 min of incubation. ${ }^{63}$ In the current study, both dseGFP/SPc complex and naked dseGFP could be taken up by Sf9 cells quickly. However, much higher fluorescent intensity was observed with dseGFP/SPc complex, and this phenomenon was more obvious after $12 \mathrm{~h}$ incubation, indicating that the SPc could promote the cellular uptake of dsRNA (Fig. 3A-B and Fig. S5). Compared to dsRNA, delivery of naked siRNA remains a considerable hurdle owing to inefficient cellular uptake. ${ }^{64}$ Saleh et al. ${ }^{65}$ demonstrated that long dsRNA bound to cells and was localized in large puncta in the cell interior, and low-level binding and no obvious internalization of siRNA were observed. As shown in Fig. S6, siRNA alone exhibited no uptake by Sf9 cells, but the cellular uptake of siRNA was remarkably improved with the help of SPc, suggesting that the SPc could efficiently deliver both long and short dsRNA across the cell membrane. Our previous study has confirmed that the SPc could combine with nucleic acids and the zeta potential of formed complex was still positive. ${ }^{49}$ The slight positive electricity of dsRNA/SPc or siRNA/SPc complex was beneficial for its adhesion to cell membrane.

Exogenous substances are commonly taken up through endocytosis, and the endocytic vesicles generated by dsRNA/nanoparticle complex travel along microtubes and subsequently fuse with early endosomes. ${ }^{27,66}$ As shown in Fig. 3C-E' and Fig. S7, the fluorescence signal was firstly located in the early vesicles close to the inner membrane of cells treated with dseGFP/SPc complex following the cellular uptake, and the complex was then dispersed into the cytoplasm quickly, suggesting that the dsRNA could be released from the vesicles. The dsRNA/SPc complex should exit endosome to avoid the degradation in late endosome (lysosome). ${ }^{67,68}$ The "proton sponge" hypothesis is the most generally accepted mechanism of endosomal escape, although it is heavily debated. ${ }^{69-71}$ Many cationic polymers have a strong buffering capacity over a range of $\mathrm{pH}$ between 5 and 7 , and the acidic environment in late endosome can lead to the protonation of their amine groups, thereby causing a water influx that leads to endosome lysis. ${ }^{27,72-73}$ Compared to naked dsRNA, there was no accumulation of SPc-delivered dsRNA in the late endosomes labelled by a specific antibody (Fig. 3F-G'), indicating that the SPc could promote the endosomal escape of dsRNA. Similar to a previous study, lipid formulated dsRNA exhibits reduced accumulation in the endosomes of Sf9 cells. ${ }^{37}$

The exocytosis and intercellular spreading of dsRNA are important for systemic RNAi, which seems to be less efficient in lepidopteran species. ${ }^{63,74}$ To directly visualize the potential exocytic vesicles of dseGFP/SPc complex, when the cellular uptake reached saturation, the culture medium containing fluorescent dseGFP/SPc complex was immediately replaced with fresh medium. The fluorescent signal was accumulated and located close to the inner membrane of cells (Fig. 3H-H' and Fig. S8), which might represent the exocytosis of the complex. Therefore, we deduced that the SPc could promote the excretion 
and intercellular spreading of dsRNA to improve systemic RNAi although we failed to detect the fluorescence signal outside the cells, which might be due to the rapid diffusion of dsRNA in culture medium. A model was further proposed to illustrate the cellular uptake and intracellular trafficking of SPCdelivered dsRNA (Fig. 3I).

\section{SPc Activates the Clathrin-Mediated Endocytosis for Enhanced dsRNA Delivery}

To identify the crucial regulators during cellular uptake of SPc-delivered dsRNA, transcriptome sequencing was performed in vitro. About $77 \%$ (116/150) of up-regulated genes and $74 \%$ (73/99) of down-regulated genes were enriched in the section of log2 (fold change) $=[0.58,1]($ Fig. 4A). The unigenes were divided into three main categories such as biological process, cellular component and molecular function (Fig. 4B). As expected, differentially expressed genes (DEGs) were enriched in various signaling pathways, and the endocytic pathway was activated by the application of dseGFP/SPC complex significantly. As crucial genes regulating endocytosis and exocytosis, AP2S1, Arf1, Rab11, CHMP5 and GRK were significantly up-regulated in cells treated with dsRNA/SPc complex (Fig. 4C). AP2S1 encodes the sigma subunit of the Adaptor Protein 2 complex, which drives endocytic vesicle

formation at the plasma membrane. ${ }^{75,76} \mathrm{ARF}$ protein regulates vesicular traffic and organelle structure by recruiting coat proteins, which plays a crucial role in fundamental biological processes, such as endocytosis, secretion, phagocytosis etc. ${ }^{77,78}$ Surprisingly, the expression of Chc gene, which encodes a major structural polypeptide of the surface lattice of clathrin-coated pits and vesicles, ${ }^{79,80}$ was not significant changed. Thus, we further performed a qRT-PCR analysis of Chc, AP2S1 and Arf1 at various incubation time points (Fig. 4D). Three genes were all up-regulated in cells treated with dsRNA/SPc complex, suggesting that the SPc might promote the cellular uptake of dsRNA by activating clathrinmediated endocytosis.

Previous studies revealed that the clathrin-mediated endocytosis might be involved in RNAi responses of Drosophila S2 cells and red flour beetles using pharmacological block. ${ }^{65,81}$ The role of clathrin-mediated endocytosis in the cellular uptake of dsRNA/SPc complex was further confirmed in vitro and in vivo using the similar method. Our results indicated that the inhibitor application hindered the cellular uptake of SPcdelivered dseGFP, and the SPc-delivered dsATP- $d$ lost biological function toward larvae fed with inhibitor, confirming that the clathrin-mediated endocytosis was the major pathway for the cellular uptake of SPcdelivered dsRNA (Fig. 5).

\section{Conclusions}

In summary, a star polymer (SPc) consisted of a hydrophilic shell with positively-charged tertiary amine in the side chain was synthesized as a gene nanocarrier, which was taken as an example to illustrate the mechanism and detailed process of nanoparticle-mediated RNAi. The SPc could assemble with dsRNA spontaneously through electrostatic force, hydrogen bond and van der Waals force. Interestingly, the SPc and another nanocarrier $\mathrm{G} 2$ could protect dsRNA from degradation by RNase A, insect hemolymph and immune cells remarkably. With the help of SPc, dsRNA could be efficiently taken up by cells, successfully 
released from early endosome, diffused into the cytoplasm, and transmitted among cells for systemic RNAi. Transcriptome analysis revealed that the SPc could up-regulate some key genes such as Chc, $A P 2 S 1$ and Arf1 for activating clathrin-mediated endocytosis pathway. Furthermore, the application of a specific inhibitor hindered the cellular uptake of dsRNA/SPc complex in vitro, and the RNAi effect was also disappeared in vivo. Our study thoroughly revealed the process how nanocarrier deliver dsRNA and this mechanism supports wide application of nanocarrier-delivered gene for therapy and pest control.

\section{Methods}

\section{Chemicals}

The SPc and PDI-cored cationic dendrimer G2 were synthesized according to the methods described by Li et al. ${ }^{49}$ and Xu et al. ${ }^{60}$, respectively. For SPc synthesis, the star initiator Pt-Br was synthesized using pentaerythritol, and the polymerization of star initiator with DMAEMA was then carried out. For G2 synthesis, the synthesis strategy was started from amine-functionalized PDI. The four primary amines selectively reacted with the acrylate group in 2-methacryloyloxyethyl acrylate to prepare the intermediate product, and the methacrylates at the periphery reacted with the thiol group in cysteamine to further prepare the $\mathrm{G} 2$.

\section{Cell Culture and Insect Rearing}

The Sf9 cells from ovaries of fall armyworms (S. frugiperda) were cultured in Sf-900 II SFM Medium (Gibco, USA) at $27^{\circ} \mathrm{C}$ supplemented with $10 \%$ fetal bovine serum, penicillin (100 unit $/ \mathrm{mL}$ ) and streptomycin $(100 \mu \mathrm{g} / \mathrm{mL})$. Larvae of $S$. frugiperda were fed on an artificial diet bought from Tuidongzhe Biotechnology Co. (China), and reared under a photoperiod of $16 \mathrm{~h} \mathrm{light:} 8 \mathrm{~h}$ dark at $25^{\circ} \mathrm{C}$.

\section{Gel Retardation Test and Isothermal Titration Calorimetry (ITC) Assays}

The enhanced green fluorescent protein gene (eGFP) was selected for dsRNA synthesis using the T7 RiboMAX expression (Promega, USA). All primers (Table S1) were synthesized by Tsingke (China). A gel retardation test was firstly performed to determine the best mass ratio for the combination of dsRNA with nanoparticle. One $\mu \mathrm{g}$ dseGFP was mixed with SPc or G2 at various mass ratios, and the mixture $(10 \mu \mathrm{L})$ was incubated at room temperature for $15 \mathrm{~min}$ and then analyzed by agarose gel electrophoresis. To determine the interaction of dsRNA with SPc, the $5.1 \mu \mathrm{M} \mathrm{SPc}$ was titrated with $0.333 \mu \mathrm{M}$ dseGFP in Nano ITC (TA Instruments Waters, USA). The heats of interaction during each injection were calculated by the integration of each titration peak via the Origin7 software (OriginLab Co., USA). The test temperature was $25^{\circ} \mathrm{C}$, and the $\Delta \mathrm{G}$ was calculated using the formula of $\Delta \mathrm{G}=\Delta \mathrm{H}-\mathrm{T} \Delta \mathrm{S}$.

\section{Stability Test of Nanocarrier-Complexed dsRNA}

To determine the stability of dsRNA, one $\mu \mathrm{g}$ dseGFP was added with RNase A (Sigma-Aldrich, USA) to prepare the reaction solution (dseGFP: $100 \mathrm{ng} / \mu \mathrm{L}$ ), and the mixture was incubated for 20 min at $37^{\circ} \mathrm{C}$. 
The hemolymph collected from $5^{\text {th }}$ instar larvae of $S$. frugiperd $a^{82}$ was diluted with $1 \times$ PBS and mixed with $1 \mu \mathrm{g}$ dseGFP, and the mixture was incubated for $1.5 \mathrm{~h}$ at room temperature. The agarose gel electrophoresis was used to detect the integrity of dsRNA treated with RNase A or hemolymph.

To investigate the stability of nanocarrier-complexed dsRNA, dsRNA was mixed with SPc and G2 at the best mass ratio, respectively. The RNase A was used to treat dseGFP/SPc or dseGFP/G2 complex (dseGFP. $1 \mu \mathrm{g}$ ). For the test of SPc-complexed dsRNA, the dseGFP/SPc complex was decomplexed in $0.3 \%$ SDS solution and analyzed using agarose gel electrophoresis. The relative band density was determined using Image J 1.48v (National Institutes of Health, USA). Meanwhile, decomplexed dseGFP was purified using MEGAclear Kit (Thermo Fisher Scientific, USA), and quantified using NanoDrop 2000 spectrophotometer (Thermo Fisher Scientific). Naked dseGFP was applied as control, and each treatment was repeated 3 times. The stability test of G2-complexed dsRNA was performed using the method described by Liu et al. ${ }^{47}$ Changes in absorption spectra of dseGFP/G2 complex treated with RNase A were detected using NanoDrop 2000 spectrophotometer. The stability of dseGFP/SPc or dseGFP/G2 complex treated with hemolymph was analyzed according to the method of dseGFP/SPc complex treated with RNase A.

For the stability test of nanocarrier-complexed dsRNA in confrontation with immune cells, $100 \mu \mathrm{L}$ of above hemolymph was added with $400 \mu \mathrm{L}$ Sf-900 II SFM Medium. Fluorescent dseGFP was synthesized, ${ }^{63}$ and then mixed with SPc and G2 at the best mass ratio, respectively. Changes in fluorescence intensity of dseGFP complexed with SPc or G2 were then tested using NanoDrop 2000 spectrophotometer. The complexation of dseGFP with $\mathrm{G} 2$ influenced the fluorescence intensity of dseGFP, and therefore SPc was used in the following experiments. The dseGFP and dseGFP/SPc complex were incubated with hemolymph for $3 \mathrm{~h}$ (dseGFP. $500 \mathrm{ng}$ ). The cells were washed, fixed with $4 \%$ paraformaldehyde, deposited on the slides using an Antifade Mounting Medium with DAPI (Vector Laboratories, USA), and then examined using a confocal microscope (Leica SP8, Germany).

\section{Cellular Uptake and Intracellular Trafficking of SPc-Delivered dsRNA/siRNA}

Fluorescent dseGFP and sieGFP (GenePharma Co., China) were used to determine the cellular uptake of SPc-delivered dsRNA/siRNA. The cells were treated with dseGFP, dseGFP/SPc complex, sieGFP and sieGFP/SPc complex, respectively (dseGFP: $500 \mathrm{ng}$; sieGFP: $280 \mathrm{ng}$ ). Cells were imaged using a confocal microscope at various time points after incubation. The time-lapse imaging for tracing dseGFP/SPc complex was conducted using an inverted fluorescence microscope (Olympus, Japan) after $1 \mathrm{~h}$ of incubation, followed by a qualification of the intracellular fluorescence intensity by Surface Plot Program using ImageJ $1.48 \mathrm{v}$ at various time points.

To investigate the intracellular trafficking of SPc-delivered dsRNA, cells incubated for $6 \mathrm{~h}$ were resuspended in fresh medium for $30 \mathrm{~min}$, and then imaged using an inverted fluorescence microscope. The intracellular fluorescence intensity was determined using Surface Plot Program similarly. Meanwhile, above cells were deposited on the slides, fixed with $4 \%$ paraformaldehyde, treated with $0.1 \%$ Triton X-100, 
and then blocked with $0.2 \%$ bovine serum albumin. Blocked samples were incubated with primary antibody mouse anti-Rab 7 (1:200, DSHB, USA) overnight, incubated with secondary antibody goat antimouse Cy5 (1:200, Jackson, USA) for $1.5 \mathrm{~h}$, and then examined using a confocal microscope.

\section{Transcriptome Analysis}

Cells were collected at $6 \mathrm{~h}$ after incubation with dseGFP and dseGFP/SPc complex (dseGFP. $500 \mathrm{ng}$ ), respectively. Total RNA was extracted from three biological replicates using the TRNzol formulation (TIANGEN, China). The transcript libraries were constructed via Illumina HiSeq sequencing platform. Raw reads containing connectors and with low-quality $(Q \leq 10)$ were removed. The resulting clean reads were assembled using Trinity software. ${ }^{83}$ TopHat2 was used to achieve the sequence alignment of clean reads with the reference genome (http://www.insect-genome.com/Sfru/) ${ }^{84}$ BLASTX was used to compare unigene sequences with the Non-Redundant protein sequence database (NR) and Swiss-Prot database for annotation of unigenes. The expression level of each transcript was presented by FPKM value. Deseq was applied for differential expression analysis between transcripts, and fold change $\geq 1.5$ and FDR $<0.05$ were screen conditions. ${ }^{85}$

The expression levels of endocytosis-related gene AP2S1, Arf and Chc were determined using quantitative real-time PCR (qRT-PCR) at various time points after the treatment of dseGFP or dseGFP/SPc complex (dseGFP: $500 \mathrm{ng}$ ). All primers for qRT-PCR were shown in Table S2. The qRT-PCR was performed with Step One Plus Real-Time PCR system (Applied Biosystems, USA) using Power SYBR® Green Master Mix (Applied Biosystems). The actin and ribosomal protein S15 (RPS-15) were selected as reference genes, and the relative mRNA levels of target genes were normalized to the abundance of two genes using the 2 $\Delta \Delta \mathrm{CT}$ methods. ${ }^{86}$

\section{Pharmacological Inhibitors of SPc-Delivered dsRNA}

Bafilomycin-A (Baf A) (MCE, USA) was used to inhibit clathrin-mediated endocytosis to determine the major pathway for SPc-mediated cellular uptake. ${ }^{65}$ For in vitro test, the cells were exposed to $0.2 \mu \mathrm{M}$ Baf A (MCE, USA) for $30 \mathrm{~min}$, treated with fluorescent dseGFP/SPc complex, deposited on the slides using an Antifade Mounting Medium with DAPI, and then examined using a confocal microscope. For in vivo test, the $V$-type proton ATPase subunit $d(A T P-d)$ was selected as a target gene, and the dsATP- $d$ was mixed with SPc and $1 \%$ volume of surfactant Alkyl Polyglucoside (Wanhua, China) to prepare the dsATP-d/SPc complex formulation (dsRNA and SPc: both $1 \mu \mathrm{g} / \mu \mathrm{L}$ ). The 2-day-old larvae of $S$. frugiperda were fed with Baf A for $2 \mathrm{~d}$, and the $0.5 \mu \mathrm{L}$ formulation was applied directly on the notum of 4-day-old larvae. The relative mRNA level of $A T P-d$ was evaluated using qRT-PCR at $24 \mathrm{~h}$ after the topical application. Each treatment consisted of 3 replications.

\section{Statistical Analysis}

The ANOVA with Tukey HSD test was conducted using SPSS 20.0 (SPSS Inc., USA) at the $P=0.05$ level of significance. A two-tailed Student's t-test was performed using Prism 7.0 (GraphPad Software Inc., USA) 
at the $P=0.05$ level of significance. The descriptive statistics were shown as the mean value and standard errors of the mean.

\section{Declarations}

\section{Acknowledgements}

This work was supported by the National Natural Science Foundation of China $(31900363,32030012$ and 32000344).

\section{Authors' contributions}

Conceptualization, SY and JS; Methodology, SY, JS, ZM and MY; Formal Analysis, SY, JS, ZM and MY; Investigation, ZM, YZ, ZC, HC and YZ; Writing-Original Draft, SY; Writing-Review \& Editing, SY, JS, ZM and MY; Funding Acquisition, SY, JS and YZ; Supervision, SY and JS.

$\mathrm{ZM}$ and $\mathrm{YZ}$ contributed equally to this work.

\section{Availability of data and materials}

The datasets used and/or analyzed during the current study are available from the corresponding author on reasonable request.

\section{Conflicts of Interest}

The authors declare no competing financial interest.

\section{Author details}

${ }^{1}$ Department of Plant Biosecurity and MOA Key Laboratory of Pest Monitoring and Green Management, College of Plant Protection, China Agricultural University, Beijing 100193, People's Republic of China.

${ }^{2}$ Institute of Plant and Environmental Protection, Beijing Academy of Agricultural and Forestry Sciences, Beijing 100097, People's Republic of China. ${ }^{3}$ College of Horticulture and Plant Protection, Yangzhou University, Yangzhou 225002, Jiangsu, People's Republic of China. ${ }^{4}$ State Key Laboratory of Chemical Resource Engineering, Beijing Lab of Biomedical Materials, Beijing University of Chemical Technology, Beijing 100029, People's Republic of China.

\section{References}

1. Fire A, Xu S, Montgomery MK, Kostas SA, Driver SE, Mello CC. Potent and specific genetic interference by double-stranded RNA in Caenorhabditis elegans. Nature. 1998;391:806-811.

2. van Rij RP, Andino R. The Silent Treatment: RNAi as a defense against virus infection in mammals. Trends Biotechnol. 2006;24:186-193. 
3. Baum JA, Bogaert T, Clinton W, Heck GR, Feldmann P, llagan O, Johnson S, Plaetinck G, Munyikwa T, Pleau $\mathrm{M}$, et al. Control of coleopteran insect pests through RNA interference. Nat. Biotechnol. 2007;25:1322-1326.

4. Zhang R, Wang B, Grossi G, Falabella P, Liu Y, Yan S, Lu J, Xi J, Wang G. Molecular basis of alarm pheromone detection in aphids. Curr. Biol. 2017;27:55-61.

5. Saw PE, Song EW. siRNA therapeutics: A clinical reality. Sci. China Life Sci. 2020;63: 485-500.

6. Zhu KY, Palli SR. Mechanisms, applications, and challenges of insect RNA interference. Annu. Rev. Entomol. 2020;65:293-311.

7. Sah DW. Therapeutic potential of RNA interference for neurological disorders. Life Sci. 2006;79:17731780.

8. Bumcrot D, Manoharan M, Koteliansky V, Sah DW. RNAi therapeutics: A potential new class of pharmaceutical drugs. Nat. Chem. Biol. 2006;2:711-719.

9. Reischl D, Zimmer A. Drug delivery of siRNA therapeutics: Potentials and limits of nanosystems. Nanomed-Nanotechnol. 2009;5:8-20.

10. Lee SJ, Kim MJ, Kwon IC, Roberts TM. Delivery strategies and potential targets for siRNA in major cancer types. Adv. Drug Deliver. Rev. 2016;104:2-15.

11. Miteva M, Kirkbride KC, Kilchrist KV, Werfel TA, Li H, Nelson CE, Gupta MK, Giorgio TD, Duvall CL. Tuning PEGylation of mixed micelles to overcome intracellular and systemic siRNA delivery barriers. Biomaterials. 2015;38:97-107.

12. Price DRG, Gatehouse JA. RNAi-meidated crop protection agaisnt insects. Trends Biotechnol. 2008;26:393-400.

13. Huvenne $H$, Smagghe G. Mechanisms of dsRNA uptake in insects and potential of RNAi for pest control: A review. J. Insect Physiol. 2010;56:227-235.

14. Zotti MJ, Smagghe G. RNAi technology for insect management and protection of beneficial insects from diseases: Lessons, challenges and risk assessments. Neotrop. Entomol. 2015;44:197-213.

15. Lü J, Guo W, Chen S, Guo M, Qiu B, Yang C, Zhang Y, Pan H. Double-stranded RNAs targeting HVRPS18 and HVRPL 13 reveal potential targets for pest management of the 28-spotted ladybeetle, Henosepilachna vigintioctopunctata. Pest Manag. Sci. 2020;76: 2663-2673.

16. Wang K, Peng Y, Pu J, Fu W, Wang J, Han Z. Variation in RNAi efficacy among insect species is attributable to dsRNA degradation in vivo. Insect Biochem. Molec. 2016;77:1-9.

17. Song H, Zhang J, Li D, Cooper AMW, Silver K, Li T, Liu X, Ma E, Zhu KY, Zhang JA. Double-stranded RNA degrading enzyme reduces the efficiency of oral RNA interfernece in migratory locust. Insect Biochem. Molec. 2017;86:68-80.

18. Guan RB, Li HC, Fan YJ, Hu SR, Christiaens O, Smagghe G, Miao XX. A nuclease specific to lepidopteran insects suppresses RNAi. J. Biol. Chem. 2018;293:6011-6021.

19. Prentice K, Smagghe G, Gheysen G, Christiaens O. Nuclease activity decreases the RNAi response in the sweetpotato weevil Cylas puncticollis. Insect Biochem. Molec. 2019;110:80-89. 
20. Cooper AM, Silver K, Zhang J, Park Y, Zhu KY. Molecular mechanisms influencing efficiency of RNA interference in insects. Pest Manag. Sci. 2019;75:18-28.

21. Guan R, Chen Q, Li H, Hu S, Miao X, Wang G, Yang B. Knockout of the HaREase Gene improves the stability of dsRNA and increases the sensitivity of Helicoverpa armigera to Bacillus thuringiensis toxin. Front. Physiol. 2019;10:1368.

22. Natarajan JV, Nugraha C, Ng XW, Venkatraman S. Sustained-release from nanocarriers: A review. J Control. Release. 2014;193:122-138.

23. Chariou PL, Ortega-Rivera OA, Steinmetz NF. Nanocarriers for the delivery of medical, veterinary, and agricultural active ingredients. ACS Nano. 2020;14:2678-2701.

24. Pardo J, Peng Z, Leblanc RM. Cancer targeting and drug delivery using carbon-based quantum dots and nanotubes. Molecules. 2018;23:378.

25. Parashar D, Rajendran V, Shukla R, Sistla R. Lipid-based nanocarriers for delivery of small interfering RNA for therapeutic use. Eur. J. Pharm. Sci. 2020;142:105159.

26. Yan S, Ren B, Zeng B, Shen J. Improving RNAi efficiency for pest control in crop species. Biotechniques. 2020;68:283-290.

27. Yan S, Ren BY, Shen J. Nanoparticle-mediated double-stranded RNA delivery system: A promising approach for sustainable pest management. Insect Sci. 2021;28:21-34.

28. Zhang X, Zhang J, Zhu KY. Chitosan/double-stranded RNA nanoparticle-mediated RNA interference to silence chitin synthase genes through larval feeding in the african malaria mosquito (Anopheles gambiae). Insect Mol. Biol. 2010;19:683-693.

29. Parsons $\mathrm{KH}$, Mondal MH, McCormick CL, Flynt AS. Guanidinium-functionalized interpolyelectrolyte complexes enabling RNAi in resistant insect pests. Biomacromolecules. 2018;19:1111-1117.

30. Das S, Debnath N, Cui Y, Unrine J, Palli SR. Chitosan, carbon quantum dot, and silica nanoparticle mediated dsRNA delivery for gene silencing in Aedes aegypti: A comparative analysis. ACS Appl. Mater. Inter. 2015;7:19530-19535.

31. Christiaens O, Tardajos MG, Reyna ZLM, Dash M, Dubruel P, Smagghe G. Increased RNAi efficacy in Spodoptera exigua via the formulation of dsRNA with guanylated polymers. Front.

Physiol. 2018;9:316.

32. Lin YH, Huang JH, Liu Y, Belles X, Lee HJ. Oral delivery of dsRNA lipoplexes to german cockroach protects dsRNA From degradation and induces RNAi response. Pest Manag. Sci. 2017;73:960-966.

33. Duncan R, Richardson SCW. Endocytosis and intracellular trafficking as gateways for nanomedicine delivery: Opportunities and challenges. Mol Pharmacol. 2012;9:2380-2402.

34. Joga MR, Zotti MJ, Smagghe G, Christiaens O. RNAi efficiency, systemic properties, and novel delivery methods for pest insect control: What we know so far. Front. Physiol. 2016;7: 553.

35. Nelemans LC, Gurevich L. Drug delivery with polymeric nanocarriers-cellular uptake mechanisms. Materials. 2020;13:366. 
36. Ramasamy T, Munusamy S, Ruttala HB, Kim JO. Smart nanocarriers for the delivery of nucleic acidbased therapeutics: A comprehensive review. Biotechnol. J. 2021;16:e1900408.

37. Gurusamy D, Mogilicherla K, Shukla JN, Palli SR. Lipids help double-stranded RNA in endosomal escape and improve RNA interference in the fall armyworm, Spodoptera frugiperda. Arch. Insect Biochem. 2020;104:e21678.

38. Hillaireau H, Couvreur P. Nanocarriers' entry into the cell: Relevance to drug delivery. Cell Mol. Life Sci. 2009;66:2873-2896.

39. Zaki NM, Tirelli N. Gateways for the intracellular access of nanocarriers: A review of receptormediated endocytosis mechanisms and of strategies in receptor targeting. Expert Opin. Drug Del. 2010;7:895-913.

40. Jhaveri A, Torchilin V. Intracellular delivery of nanocarriers and targeting to subcellular organelles. Expert Opin. Drug Del. 2016;13:49-70.

41. Ehrlich M, Boll W, van Oijen A, Hariharan R, Chandran K, Nibert ML, Kirchhausen T. Endocytosis by random initiation and stabilization of clathrin-coated pits. Cell. 2004;118:591-605.

42. Doherty GJ, McMahon HT. Mechanisms of endocytosis. Annu. Rev. Biochem. 2009;78:857-902.

43. Shete HK, Prabhu RH, Patravale VB. Endosomal escape: A bottleneck in intracellular delivery. J. Nanosci. Nanotechnol. 2014;14:460-474.

44. He B, Chu Y, Yin M, Müllen K, An C, Shen J. Fluorescent nanoparticle delivered dsRNA toward genetic control of insect pests. Adv. Mater. 2013;25:4580-4584.

45. Zheng Y, Hu Y, Yan S, Zhou H, Song D, Yin M, Shen J. A polymer/detergent formulation improves dsRNA penetration through the body wall and RNAi-induced mortality in the soybean aphid Aphis glycines. Pest Manag. Sci. 2019;75:1993-1999.

46. Liu X, Zheng Y, Zhang S, Liu K, Zhang S, Yin M, Zhang L, Shen J. Perylenediimide-cored cationic nanocarriers deliver virus DNA to kill insect pests. Polym. Chem. 2016;7:3740-3746.

47. Liu X, He B, Xu Z, Yin M, Yang W, Zhang H, Cao J, Shen J. A functionalized fluorescent dendrimer as a pesticide nanocarrier: Application in pest control. Nanoscale. 2015;7:445-449.

48. Zheng Y, You S, Ji C, Yin M, Yang W, Shen J. Development of an amino acid-functionalized fluorescent nanocarrier to deliver a toxin to kill insect pests. Adv. Mater. 2016;28:1375-1380.

49. Li J, Qian J, Xu Y, Yan S, Shen J, Yin M. A facile-synthesized star polycation constructed as a highly efficient gene vector in pest management. ACS Sustain. Chem. Eng. 2019;7:6316-6322.

50. Yan S, Qian J, Cai C, Ma Z, Li J, Yin M, Ren B, Shen J. Spray method application of transdermal dsRNA delivery system for efficient gene silencing and pest control on soybean aphid Aphis glycines. J. Pest Sci. 2019;93:449-459.

51. Guo S, Guo X, Zheng L, Zhao Z, Liu L, Shen J, Li Z. A potential genetic control by suppression of the wing developemental gene wingless in a alobal invasive pest Bactrocera dorsalis. J. Pest Sci. 2021;94:517-529. 
52. Wei H, Tan S, Yan S, Li Z, Shen J, Liu X. Nanocarrier-mediated transdermal dsRNA-NPF1 delivery system contributes to pest control via inhibiting feeding behavior in Grapholita molesta. J. Pest Sci. 2021. https://doi.org/10.1007/s10340-021-01422-y.

53. Zhang YH, Ma ZZ, Zhou H, Chao ZJ, Yan S, Shen J. Nanocarrier-delivered dsRNA suppresses wing development of green peach aphids. Insect Sci. 2021. https://doi.org/10.1111/1744-7917.12953.

54. Ross PD, Subramanian S. Thermodynamics of protein association reactions: Forces contributing to stability. Biochemistry. 1981;20:3096-3102.

55. Yan S, Hu Q, Li J, Chao Z, Cai C, Yin M, Du X, Shen J. A star polycation acts as a drug nanocarrier to improve the toxicity and persistence of botanical pesticides. ACS Sustain. Chem. Eng. 2019;7:1740617413.

56. Yan S, Hu Q, Jiang Q, Chen H, Wei J, Yin M, Du X, Shen J. Simple osthole/nanocarrier pesticide efficiently controls both pests and diseases fulfilling the need of green production of strawberry. ACS Appl. Mater. Inter. 2021;13:36350-36360.

57. Yan S, Cheng WY, Han ZH, Wang D, Yin MZ, Du XG, Shen J. Nanometerization of thiamethoxam by a cationic star polymer nanocarrier efficiently enhances the contact and plant-uptake dependent stomach toxicity against green peach aphids. Pest Manag. Sci. 2021; 77:1954-1962.

58. Wang X, Zheng K, Cheng W, Li J, Liang X, Shen J, Dou D, Yin M, Yan S. Field application of star polymer-delivered chitosan to amplify plant defense against potato late blight. Chem. Eng. J. 2021;417:129327.

59. Garbutt JS, Bellés X, Richards EH, Reynolds SE. Persistence of double-stranded RNA in insect hemolymph as a potential determiner of RNA interference success: Evidence from Manduca sexta and Blattella germanica. J. Insect Physiol. 2013;59:171-178.

60. Xu Z, He B, Shen J, Yang W, Yin M. Fluorescent water-soluble perylenediimide-cored cationic dendrimers: Synthesis, optical properties, and cell uptake. Chem. Commun. 2013;49:3646-3648.

61. Bell JK, Askins J, Hall PR, Davies DR, Segal DM. The dsRNA binding site of human toll-like receptor 3. P. Natl. Acad. Sci. USA. 2006;103:8792-8797.

62. Peisley A, Hur S. Multi-level regulation of cellular recognition of viral dsRNA. Cell. Mol. Life Sci. 2013;70:1949-1963.

63. Shukla JN, Kalsi M, Sethi A, Narva KE, Fishilevich E, Singh S, Mogilicherla K, Palli SR. Reduced stability and intracellular transport of dsRNA contribute to poor RNAi response in lepidopteran insects. RNA Biol. 2016;13:656-669.

64. Kesharwani P, Gajbhiye V, Jain NK. A review of nanocarriers for the delivery of small interfering RNA. Biomaterials. 2012;33:7138-7150.

65. Saleh MC, Van Rij RP, Hekele A, Gillis A, Foley E, O'Farrell PH, Andino R. The endocytic pathway mediates cell entry of dsRNA to induce RNAi silencing. Nat. Cell Biol. 2006;8:793-802.

66. Cappelle K, de Oliveira CFR, van Eynde B, Christiaens O, Smagghe G. The involvement of clathrinmediated endocytosis and two sid-1-like transmembrane proteins in double-stranded RNA uptake in the colorado potato beetle midgut. Insect Mol. Biol. 2016;25:315-323. 
67. Di Guglielmo GM, Le Roy C, Goodfellow AF, Wrana JL. Distinct endocytic pathways regulate TGF$\beta$ receptor signaling and turnover. Nat. Cell Biol. 2003;5:410-421.

68. Varkouhi AK, Scholte M, Storm G, Haisma HJ. Endosomal escape pathways for delivery of biologicals. J. Control. Release. 2011;151:220-228.

69. Akinc A, Thomas M, Klibanov AM, Langer R. Exploring polyethylenimine-mediated DNA transfection and the proton sponge hypothesis. J. Gene Med. 2005;7:657-663.

70. Benjaminsen RV, Mattebjerg MA, Henriksen JR, Moghimi SM, Andresen TL. The possible "proton sponge" effect of polyethylenimine (PEI) does not include change in lysosomal PH. Mol. Ther. 2013;21:149-157.

71. Vermeulen LMP, De Smedt SC, Remaut K, Braeckmans K. The proton sponge hypothesis: Fable or fact? Eur. J. Pharm. Biopharm. 2018;129:184-190.

72. Boussif O, Lezoualc'h F, Zanta MA, Mergny MD, Scherman D, Demeneix B, Behr JP. A versatile vector for gene and oligonucleotide transfer into cells in culture and in vivo: Polyethylenimine. P. Natl. Acad. Sci. USA. 1995;92:7297-7301.

73. Sonawane ND, Szoka JrFC, Verkman AS. Chloride accumulation and swelling in endosomes enhances DNA transfer by polyamine-DNA polyplexes. J. Biol. Chem. 2003;278:44826-44831.

74. Terenius O, Papanicolaou A, Garbutt JS, Eleftherianos I, Huvenne H, Kanginakudru S, Albrechtsen M, An C, Aymeric JL, Barthel A, Bebas P, et al. RNA Interference in Lepidoptera: An Overview of Successful and Unsuccessful Studies and Implications for Experimental Design. J. Insect Physiol. 2011;57:231-245.

75. Schmid SL. Clathrin-coated vesicle formation and protein sorting: An integrated process. Annu. Rev. Biochem. 1997;66:511-548.

76. Nesbit MA, Hannan FM, Howles SA, Reed AAC, Cranston T, Thakker CE, Gregory L, Rimmer AJ, Rust N, Graham U, Morrison PJ, et al. Mutations in AP2S1 Cause Familial Hypocalciuric Hypercalcemia Type 3. Nat. Genet. 2013;45:93-97.

77. D'Souza-Schorey C, Chavrier P. ARF proteins: Roles in membrane traffic and beyond. Nat. Rev. Mol. Cell Biol. 2006;7:347-358.

78. Adarska P, Wong-Dilworth L, Bottanelli F. ARF GTPases and their ubiquitous role in intracellular trafficking beyond the Golgi. Front. Cell Dev. Biol. 2021;9:679046.

79. O'Halloran TJ, Anderson RG. Clathrin heavy is required for pinocytosis, the presence of large vacuoles, and development in dictyostelium. J. Cell Biol. 1992;118:1371-1377.

80. Bazinet C, Katzen AL, Morgan M, Mahowald AP, Lemmon SK. The Drosophila clathrin heavy chain gene: clathrin function is essential in a multicellular organism. Genetics. 1993;134:1119-1134.

81. Xiao D, Gao X, Xu J, Liang X, Li Q, Yao J, Zhu KY. Clathrin-dependent endocytosis plays a predominant role in cellular uptake of double-stranded RNA in the red flour beetle. Insect Biochem. Mol. Biol. 2015;60:68-77. 
82. Singh IK, Singh S, Mogilicherla K, Shukla JN, Palli SR. Comparative analysis of double-stranded RNA degradation and processing in insects. Sci. Rep. 2017;7:17059.

83. Grabherr MG, Haas BJ, Yassour M, Levin JZ, Thompson DA, Amit I, Adiconis X, Fan L, Raychowdhury $\mathrm{R}$, Zeng $\mathrm{Q}$, et al. Full-length transcriptome assembly from RNA-Seq data without a reference genome. Nat. Biotechnol. 2011;29:644-652.

84. Kim D, Pertea G, Trapnell C, Pimentel H, Kelley R, Salzberg SL. TopHat2: Accurate alignment of transcriptomes in the presence of insertions, deletions and gene fusions. Genome Biol. 2013;14:R36.

85. Love MI, Huber W, Anders S. Moderated estimation of fold change and dispersion for RNA-Seq data with DESeq2. Genome Biol. 2014;15:550.

86. Livak KJ, Schmittgen TD. Analysis of relative gene expression data using real-time quantitative PCR and the $2^{-\Delta \Delta C T}$ method. Methods. 2001;25:402-408.

\section{Figures}
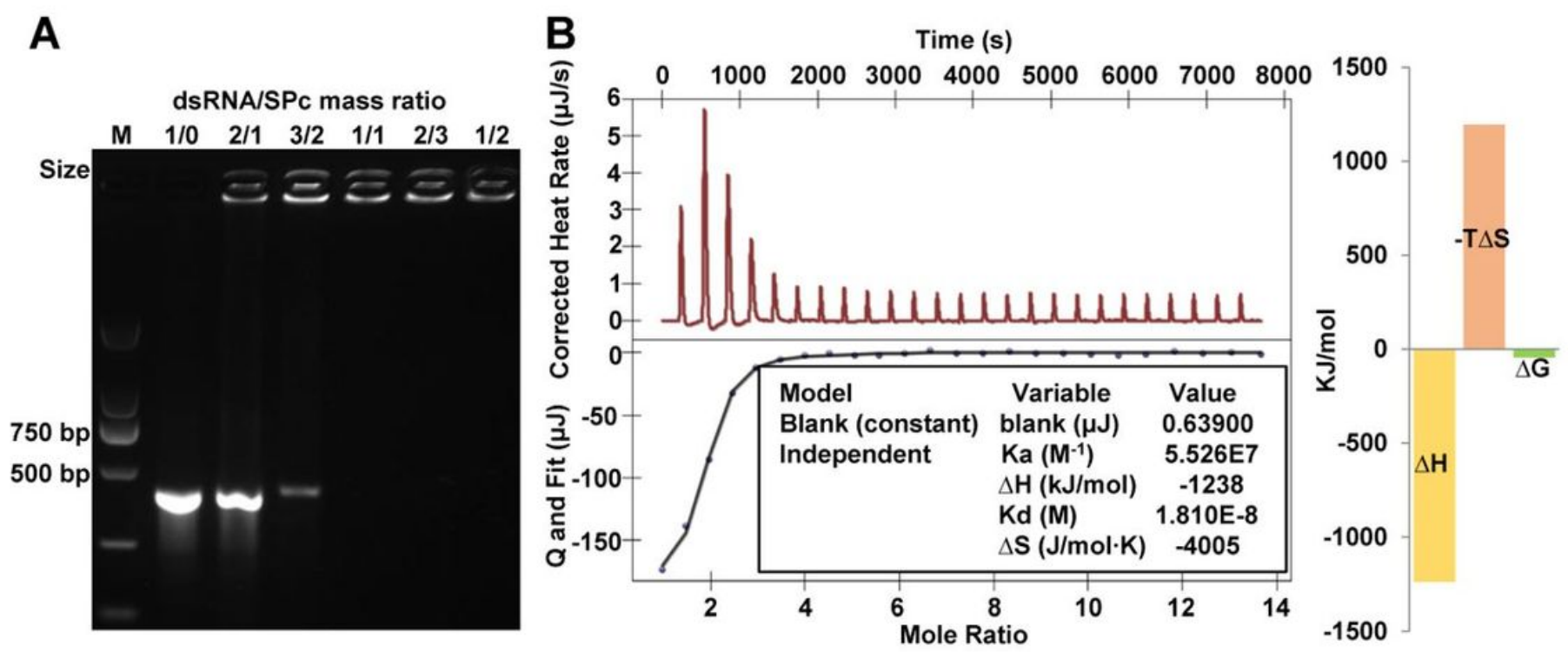

\section{Figure 1}

Self-assembly mechanism of dsRNA/SPc complex. (A) Gel electrophoresis assay of dseGFP retardation by SPc at various mass ratios. M: DNA marker. (B) ITC titration of dseGFP $(0.333 \mu \mathrm{M})$ into SPc solution $(5.1 \mu \mathrm{M})$. 

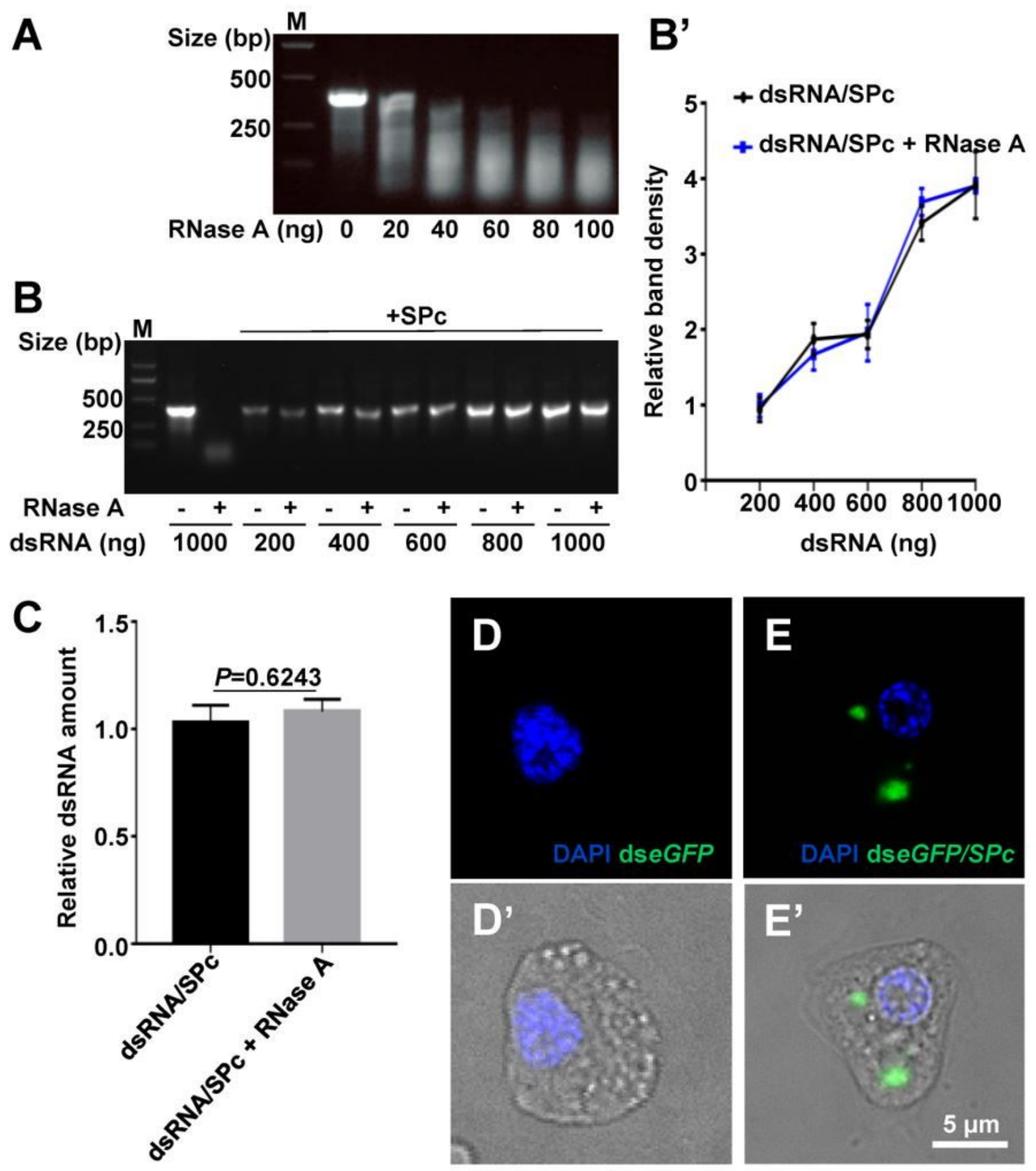

Figure 2

Enhanced stability of SPc-complexed dsRNA. (A) The dseGFP degradation by RNase A. M: DNA marker. (B-B') Gel electrophoresis assay (B) and relative band density ( $\left.\mathrm{B}^{\prime}\right)$ of SPc-complexed dseGFP treated with RNase A. (C) Relative dsRNA amount of SPc-complexed dseGFP treated with RNase A. Statistical analysis was conducted using independent $t$-test at the $P=0.05$ level of significance. (D-E') Stability test of naked dseGFP(D-D') or SPc-complexed dseGFP (E-E') treated with immune cells. Blue: DAPI. Green: dseGFP. 

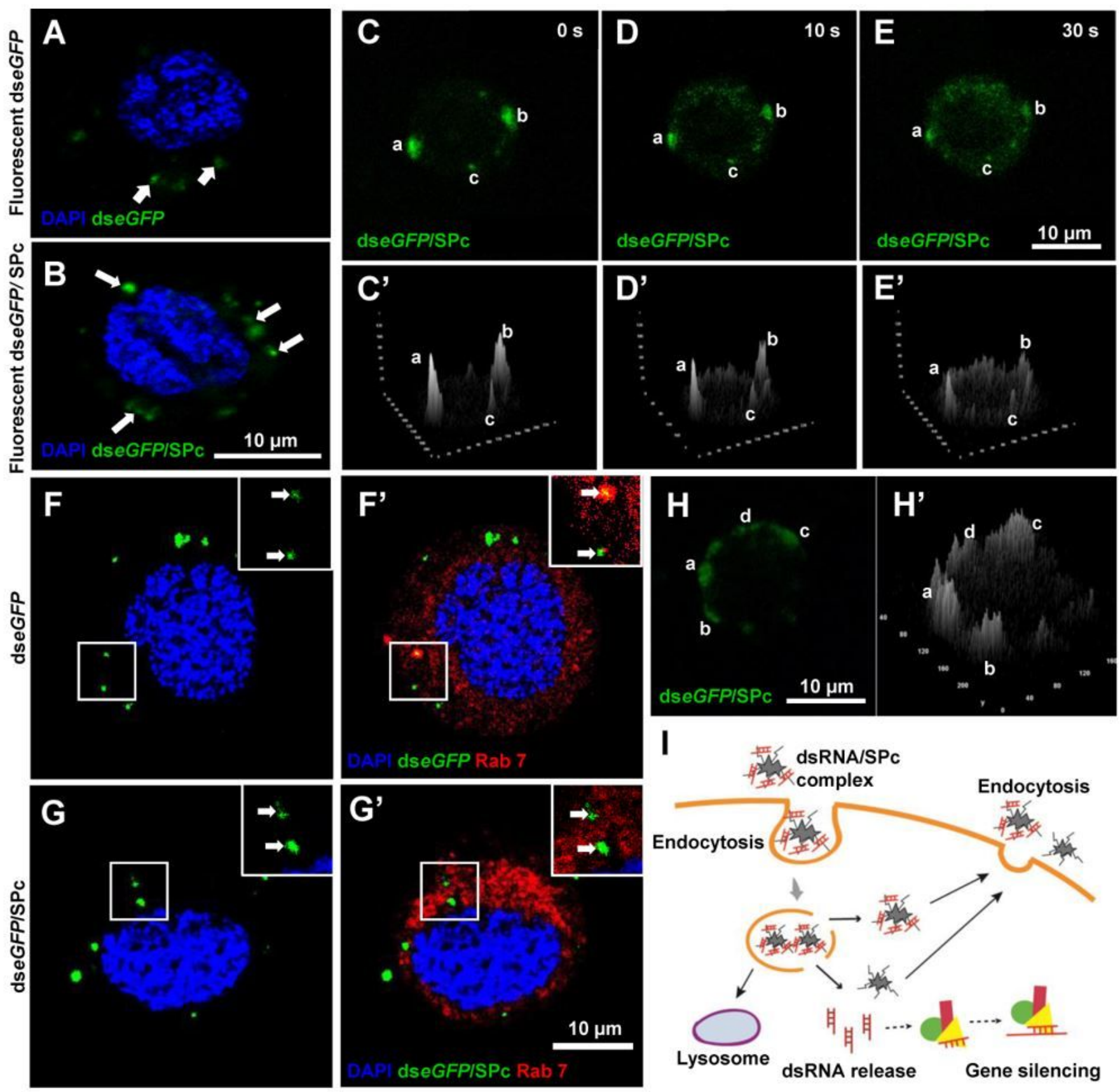

\section{Figure 3}

Improved endocytosis and exocytosis of dsRNA/SPc complex. (A-B) Cellular uptake of naked dseGFP(A) and dseGFP/SPc complex (B). Blue: DAPI. Green: dseGFP. (C-E') Vesicle release of SPc-delivered dseGFP into cytoplasm. A surface plot test was conducted using ImageJ. Three typical regions were marked. (F$\left.\mathrm{G}^{\prime}\right)$ Endosomal escape of SPc-delivered dsRNA. Red: Rab7 marking the late endosome. (H-H') Potential exocytosis of dsRNA/SPc complex. The dseGFP/SPc complex was accumulated and located close to the inner membrane of cells. (I) Schematic illustration of cellular uptake and intracellular trafficking of SPcdelivered dsRNA. 
A

\begin{tabular}{lccc}
\hline DEG set & ALL DEG & Up-regulated & Down-regulated \\
\hline $\begin{array}{l}\text { dseGFPISPc } \\
\text { vs dseGFP }\end{array}$ & 249 & 150 & 99 \\
\hline
\end{tabular}

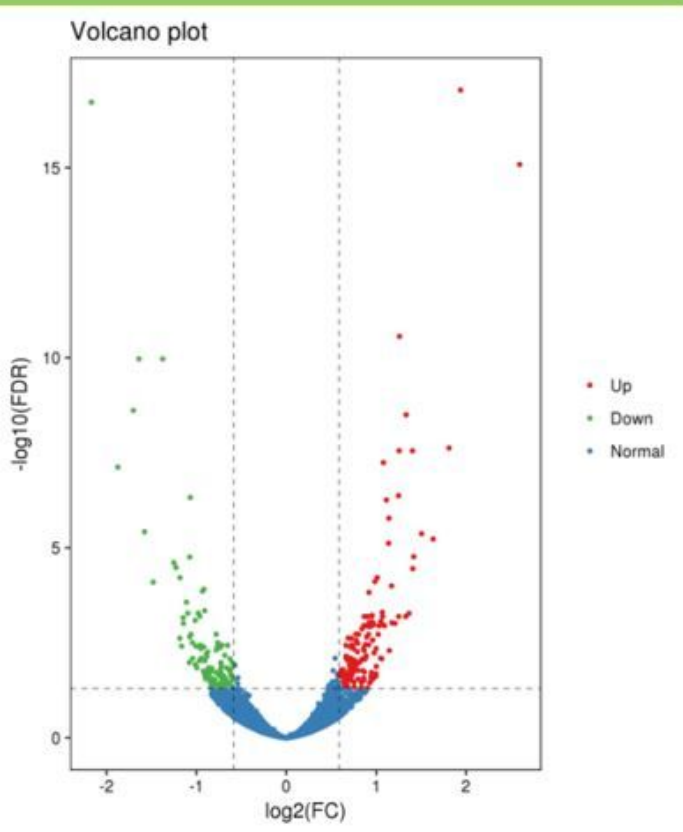

C

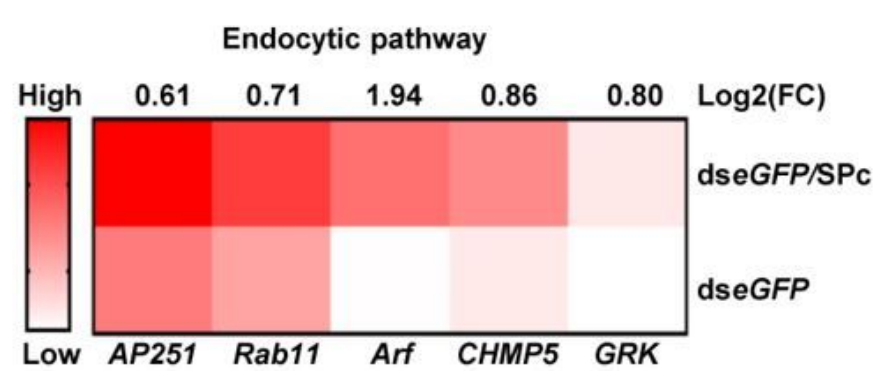

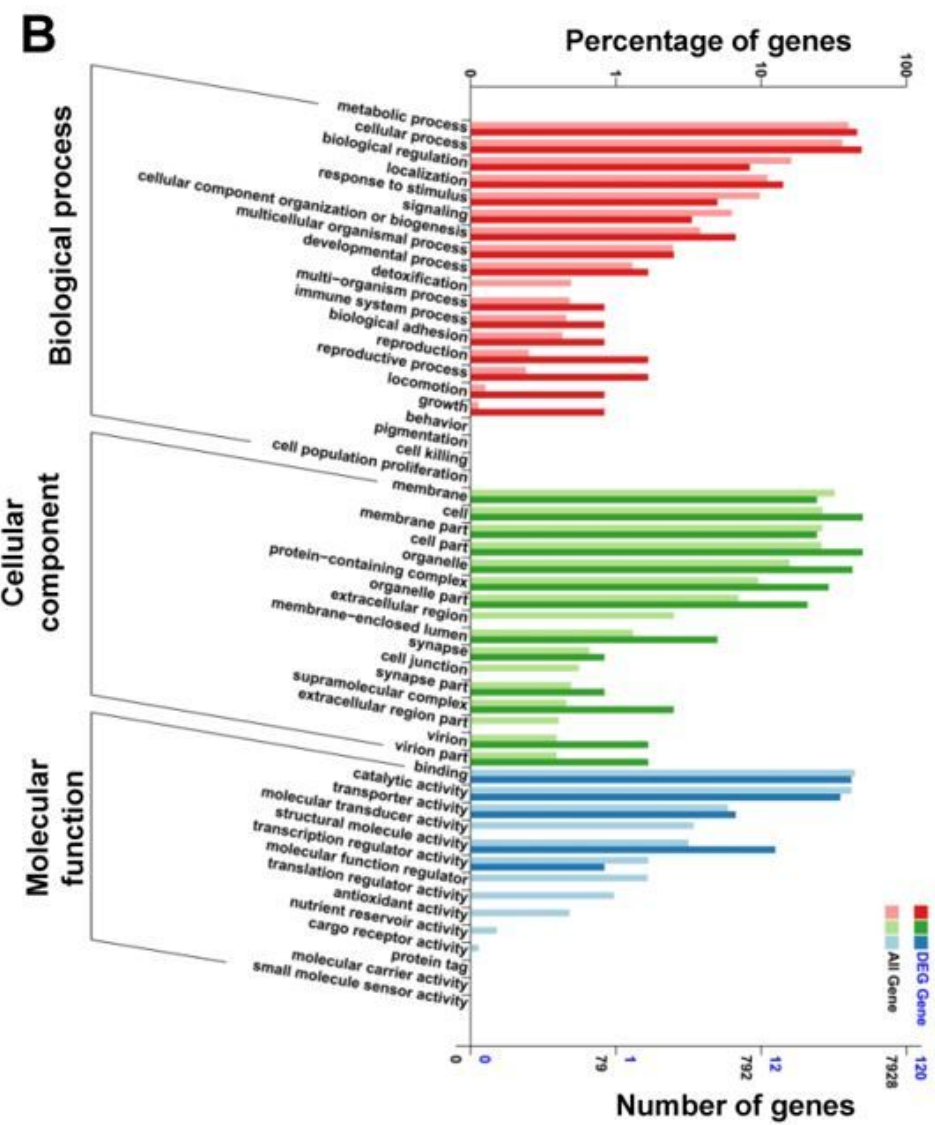

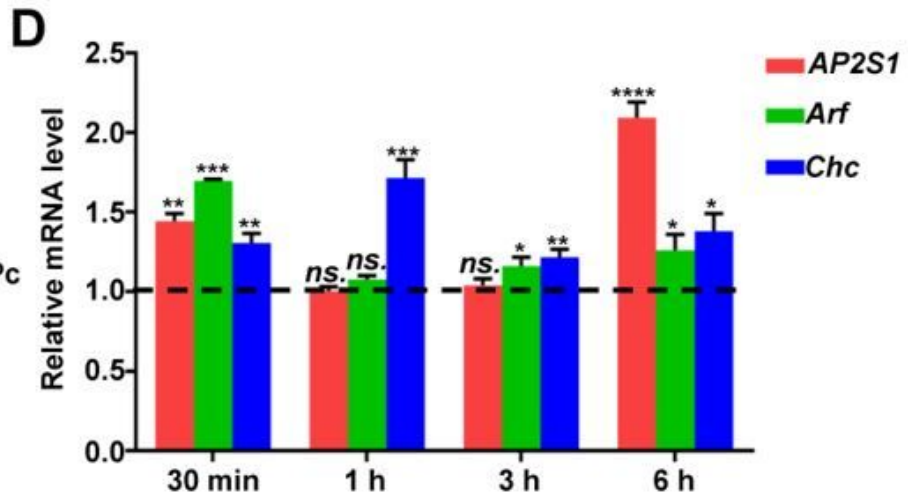

\section{Figure 4}

Comparative transcriptome analysis of Sf9 cells treated with naked dseGFP and dseGFP/SPc complex. (A-B) Analysis of differentially expressed genes (DEGs) with volcano plot (A) and GO annotation (B). (C) Heatmap of the endocytic pathway. (D) QRT-PCR analysis of the genes related to clathrin-mediated endocytosis. Asterisk indicates significant difference in gene expression between dseGFP/SPc complex and naked dseGFP treatment according to independent $t$-test (ns.: no significant difference, ${ }^{\star} P<0.05$, $\star \star P<0.01, * \star \star P<0.001$ and $* \star \star \star P<0.0001)$. 


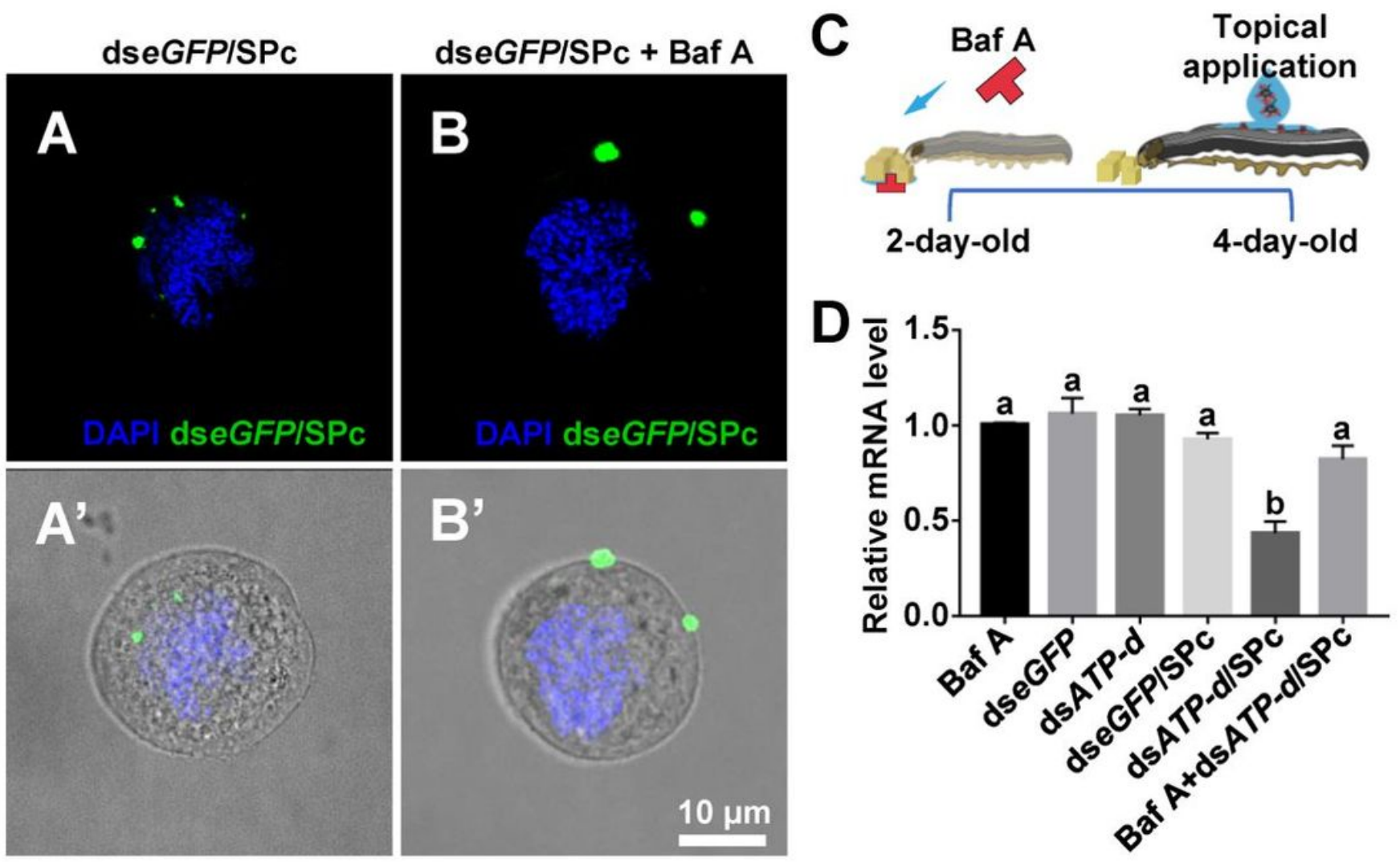

Figure 5

Pharmacological inhibitor blocks the cellular uptake of dsRNA/SPc complex. (A-B') Inhibitor application hindered the cellular uptake of dseGFP/SPc complex. Blue: DAPI. Green: dseGFP. (C) Schematic diagram of pharmacological assay toward $S$. frugiperda. The 2-day-old larvae were fed with Baf $A$ for 2 days. Then, RNAi was performed using 4-d-old larvae. (D) QRT-PCR analysis of ATP-d gene expression in larvae fed with inhibitor. Different letters on columns indicate significant differences (Tukey HSD test, $P<0.05$ ).

\section{Supplementary Files}

This is a list of supplementary files associated with this preprint. Click to download.

- Graphicabstract.pdf

- Additionalfle1.docx

- Additionalfle2.docx 